\title{
Analyser le travail conjoint entre différents systèmes d'activité
}

Analysing joint work between activity systems

Analizando el trabajo conjunto entre sistemas de actividades

\section{Christine Owen}

\section{(Q) OpenEdition}

Journals

Édition électronique

URL : http://journals.openedition.org/activites/2053

DOI : 10.4000/activites.2053

ISSN : 1765-2723

Éditeur

ARPACT - Association Recherches et Pratiques sur les ACTivités

\section{Référence électronique}

Christine Owen, "Analyser le travail conjoint entre différents systèmes d'activité », Activités [En ligne], 5-2 I octobre 2008, mis en ligne le 15 octobre 2008, consulté le 19 avril 2019. URL : http:// journals.openedition.org/activites/2053 ; DOI : 10.4000/activites.2053

\section{(c) (i) (2)}

Activités est mis à disposition selon les termes de la licence Creative Commons Attribution - Pas d'Utilisation Commerciale - Pas de Modification 4.0 International. 


\title{
Analyser le travail conjoint entre différents systèmes d'activité
}

\author{
Christine Owen \\ Faculté d'éducation, Université de Tasmanie \\ Private Bag 66, Hobart TAS 7001 \\ Christine.Owen@utas.edu.au
}

\begin{abstract}
This paper aims to contribute to the development of third-generation activity theorizing. It does so by analysing (inter)acting subjects engaged in joint work at the borders of their respective activity systems. The paper explores these issues theoretically by discussing practices observed in pilot-controller interaction in the aviation industry. In this case the way in which work practices are jointly mediated through changes occurring in interacting activity systems are examined. The analysis will show how the changes in elements of the activity systems reveal points of tension and contestation and thus opportunities for development in the everyday interactions between air traffic controllers and airline pilots. In undertaking the analysis, the paper introduces some key questions for consideration when designing interventions in such work environments and contributes to the development of third-generation activity theory.
\end{abstract}

KEY WORDS :

Cultural-historical activity theory, air traffic control/aircrew coordination, collaboration, organisational theory.

\section{Introduction}

Dans un article publié par ce journal, Virkkunen (2006) s'intéressait aux contradictions rencontrées durant des changements organisationnels destinés à créer des pratiques de travail plus efficaces. Il notait alors que la transformation de l'activité implique souvent des formes de collaboration qui franchissent les frontières organisationnelles établies. Sur cette base, il examinait comment les acteurs se heurtent à des choix, qui gênent à la fois l'identification des changements et leur maintien. Je partage l'objectif de Virkkunen qui est d'améliorer les pratiques de travail, et sa préoccupation visant à rendre visible les pratiques professionnelles. Dans cet article, je m'intéresse à la façon dont des sujets agissants s'engagent dans une interaction située aux frontières de leurs systèmes d'activité, et à ce qui permet ou limite leur collaboration.

Mon analyse vise à développer un cadre pouvant être utilisé par des chercheurs et des praticiens pour étudier ce qui permet et limite une collaboration inter organisationnelle, en mettant au jour les tensions qui surviennent aux frontières de systèmes d'activité interdépendants (Engeström, Engeström, \& Karkkainen 1995).

\section{Le travail conjoint entre des systèmes d'activité interdépendants}

On a beaucoup souligné que le travail est devenu de plus en plus intense, complexe et interdépendant. En partie, ceci a pour origine le développement d'une économie de la connaissance et de la globalisation, et de changement dans les aménagements du travail avec pour résultat des structures 
organisationnelles toujours plus monotones, et des technologies de l'information et de la communication capables d'ajouter des niveaux d'interconnectivité qui augmentent la demande de réactivité, à la fois au sein des organisations et entre elles (Casey, 1999 ; Gerber, \& Lanksherar, 2000 ; Rouilleault, 2000 ; Boreham, Samurçay, \& Fischer, 2002 ; Engeström, 2005 ). Ces changements entraînent l'augmentation des échecs et des possibilités d'échec, avec parfois des conséquences considérables (Woods, 2006). Les problèmes que soulèvent ces changements sont que les ouvriers travaillent de plus en plus dans des environnements caractérisés par:

- des conditions dynamiques en constantes évolutions;

- une interdépendance des tâches impliquant des agents multiples;

- une intensification du travail qui conduit à un besoin toujours plus grand de réactivité dans des délais toujours plus courts;

- et un travail rendu plus abstrait par l'utilisation de ressources informationnelles, lesquels impliquent l'interprétation de symboles et exigent une activité plus cognitive.

L'industrie aéronautique illustre bien ces dimensions, en particulier pour ce qui est du travail conjoint entre les pilotes d'avion et les contrôleurs au sol qui gèrent la circulation du trafic aérien. Dans l'industrie aéronautique, les problèmes ont surgi suite à l'augmentation de la demande de transports aériens qui a conduit à une multiplication des vols et à une augmentation de la densité des avions au sein de l'espace aérien. Ce qui entraîne une réduction de l'espace qui sépare les avions. La montée en flèche des coûts de carburant et de maintenance a ajouté une pression supplémentaire, à la fois sur les pilotes et sur les contrôleurs qui doivent continuer à fournir un service efficace. Ceci s'ajoute à la sûreté des vols qui est la préoccupation majeure des opérateurs. Pour répondre à ces demandes, les développements technologiques ont porté sur l'interopérabilité, créant une interdépendance toujours plus étroite entre l'équipage et les contrôleurs. Ces développements représentent un défi cognitif et de communication considérable pour les opérateurs impliqués.

Dans cet article, je souhaite montrer qu'il est important de mieux comprendre ce qui permet et limite la réalisation d'un travail conjoint, en particulier dans des domaines comme l'aviation.

L'objectif de ce papier est double. Premièrement, il vise à proposer une interprétation renouvelée de la collaboration entre l'équipage et les contrôleurs aériens, en analysant cette action comme un travail conjoint, situé aux frontières de deux systèmes d'activité en interaction. Deuxièmement, il souhaite contribuer au développement de ce que Engeström (2006) appelle la troisième génération des théories de l'activité. D'après Engeström (2000b; 2004), une troisième génération des théories de l'activité est nécessaire pour mieux appréhender les intersections entre des systèmes d'activité mobilisés dans des réseaux distribués. Engeström développe cette idée à l'aide de trois concepts principaux: le « boundary crossing ${ }^{l} »$ (Engeström, Engeström, \& Karkkainen, 1995); le « travail en nœud» (knotworking, Ahonen, Engeström, \& Virkkunen 2000) et la « co-configuration » (co-configuration, Engeström, 2000a, 2004). Dans ce papier, je contribuerai à ce développement en employant des concepts issus de la théorie des organisations, afin d'aider à comprendre les pratiques de travail conjoint situées à la frontière des systèmes d'activité en interaction et ce faisant, j'espère également apporter ma contribution à la théorisation de l'activité de troisième génération.

Pour commencer, il est d'abord nécessaire de situer la trajectoire développementale du travail conjoint des contrôleurs aérien et de l'équipage des avions dans son contexte, grâce à un survol rapide des recherches déjà menées.

\section{L'interdépendance du travail de l'équipage et des contrôleurs aériens}

L'interdépendance est inhérente au travail de l'équipage et des contrôleurs aériens. Le travail du

1 En français le terme « boundary crossing » (littéralement « franchissement de frontière ») est quasiment intraduisible On a préféré conserver la locution anglaise (NdT). 
pilote ne peut être correctement accompli sans le contrôleur aérien, tout au moins pas dans sa forme actuelle. Et sans le pilote, le travail du contrôleur aérien ne pourrait pas exister. Pourtant, l'accomplissement conjoint de ce travail soulève de nombreux défis.

Le premier est que le travail entre les pilotes et les contrôleurs du trafic aérien s'effectue dans un espace virtuel. En effet, malgré leur travail conjoint et interdépendant, ils ne sont jamais co-présents. L'espace de travail du pilote est le poste de pilotage de l'avion et l'espace de travail du contrôleur est le centre de contrôle au sol. Une grande partie du travail de communication des sujets agissants entre ces deux espaces de travail s'effectue par radio ainsi que par l'intermédiaire de messages semblables aux e-mails lors de certaines phases du vol (connus sous le terme de controller-pilot data link communication). Toutefois, chacun d'eux est engagé dans l'accomplissement conjoint du travail. Ils partagent une finalité mutuelle et se tiennent mutuellement responsables des aspects particuliers de ce travail. Ils partagent un langage, des routines et des artefacts. Par ailleurs, afin de gérer correctement un vol, ils doivent se faire confiance. Les pilotes confient littéralement leur vie et celle de leurs passagers à la voix de contrôleurs invisibles. Les contrôleurs, qui dirigent plusieurs avions contenant souvent des centaines de passagers chacun, attendent des pilotes qu'ils suivent leurs instructions au sein du volume limité d'un espace aérien donné.

Pendant plus de 25 ans, les chercheurs ont analysé les facteurs humains et explorés différentes approches pour tenter de comprendre ce qui provoque des erreurs et améliorer la sécurité de ces environnements (Hollnagel, \& Woods, 2006). Pourtant, même depuis récemment, la littérature sur les facteurs humains dans l'aviation est restée largement focalisée sur les systèmes technologiques (Field, \& Harris, 1998; McGann, Morrow, Rodvold, \& Mackintosh, 1998; Dekker, 2000; Prinzo, 2004; Rantanen, McCarley, \& Xu, 2004) ou sur les ressources cognitives de traitement de l'information et de la communication (Cushing, 1995; Fowler, 1999; Salas, Bowers, \& Edens, 2001; Yule, Flin, \& Murdy, 2007).

$\mathrm{Au}$ fur à mesure que les technologies et les systèmes sont devenus de plus en plus interconnectés, l'attention s'est portée sur le développement de systèmes interopérables et sur l'étude des conséquences de ces systèmes sur les rôles liés aux places respectives des acteurs (McCann, Baranski, Thompson, \& Pigeau, 2000; Smith-Jentsch, Baker, Salas, \& Cannon-Bowers, 2001). En fait, dans les années 90, l'intégration des systèmes équipage/contrôle au sol était considérée, dans la littérature des facteurs humains aéronautiques, comme le plus grand défi du 21 ème siècle. Toutefois, intégrer des technologies destinées à prendre en charge l'activité du travail entre l'équipage et le poste de contrôle n'a pas été une tâche facile. Kerns (1999, p. 520), par exemple, avait remarqué que les systèmes technologiques qui prennent en charge l'activité de travail de l'équipage et du contrôle ont été historiquement construits pour " interagir au sein de leurs systèmes respectifs, et non pas entre eux » (italique ajouté). Ceci était dû, selon Kerns (ibid.), au fait que «la conception des systèmes orientés sol et des systèmes orientés air était régie par une technologie et des objectifs organisationnels internes, et que ni les procédures, ni les outils n'étaient bien adaptés à l'usage coordonné des contrôleurs et des pilotes dans le but d'accomplir des objectifs communs ». Il faut dire que le problème d'interopérabilité entre les systèmes et leurs opérateurs n'affecte pas uniquement le domaine aéronautique, c'est une problématique qui va devenir de plus en plus cruciale du fait de l'augmentation de l'interconnectivité (Howitt, \& Leonard, 2006).

La recherche a aussi tenté de savoir comment et pourquoi les ruptures de la communication entrâ̂nant des accidents mortels peuvent survenir entre des personnels apparemment surentraînés et techniquement très compétents (Loftus, 1979; Wiener, Kanki, \& Helmreich, 1993). Selon la littérature traitant des facteurs humains, littérature qui est restée prolifique, il est important pour renforcer la sécurité, d'améliorer l'accessibilité aux ressources de communication par le personnel de l'aviation par l'intermédiaire du travail en équipe. Une grande partie de cette recherche s'appuie sur les équipes, notamment celle composée de l'équipage (Kanki, \& Palmer, 1993; Morrow, Rodvold, \& Lee, 1994 ; Orlady, \& Orlady, 1999; Salas et. al., 2001; Salas, Stagl, \& Burke, 2004) et du contrôle du trafic aérien (Hartel, \& Hartel, 1995; Smith-Jentsch et al., 2001). Sur la base de cette littérature, l'attention 
s'est portée sur la compréhension des raisons pour lesquelles la conscience de la situation entre les parties peut être en échec (Jentsch, Barnett, Bowers, \& Salas, 1999), sur le type de stratégies verbales et non-verbales que les membres peuvent utiliser pour maintenir une représentation cognitive partagée (Segal, 1994), et sur le type de stratégies qu'un formateur peut employer pour encourager les membres à créer le modèle mental d'une équipe (Salas et al, 2001; Salas et al., 2004).

Ces recherches apportent une forme de compréhension, mais elles omettent deux considérations importantes. Premièrement, en regardant de plus près, il est difficile d'affirmer que le travail conjoint entre le contrôleur et l'équipage est un travail d'équipe, dans le sens où le terme d'équipe est habituellement défini. On peut définir le travail en équipe comme: un ensemble distinct de deux ou plusieurs personnes qui interagissent de façon dynamique, interdépendante et adaptée en vue d'un objectif, d'un but ou d'une mission commune et valorisée, qui se voient chacune assignée d'un rôle ou d'une fonction à exécuter, et dont la durée d'appartenance est limitée (Baker, \& Salas, 1992 p. 469). Et le travail entre équipage et contrôle au sol remplit ces critères. Mais pourtant rares sont les pilotes et les contrôleurs à penser qu'ils forment une équipe, en particulier parce que la durée d'appartenance à cette équipe est extrêmement ténue. Les indicateurs mesurant habituellement l'efficacité d'une équipe (par exemple la cohésion) ne s'appliquent pas ici.

Deuxièmement, l'accent mis sur les systèmes ou sur les processus de communication ne rend pas compte de manière satisfaisante de ce qui permet et limite les pratiques conjointes réussies. Il s'agit d'une omission capitale. Je souhaite argumenter qu'il est nécessaire d'avoir une compréhension exhaustive du développement d'une activité de travail conjointe en analysant les tensions et les contradictions inhérentes au travail, par l'étude des processus et des structures sous-jacentes. Pour cela, il faut appréhender le travail comme celui de deux systèmes d'activités interdépendants en interaction. Afin de développer cette idée, je vais d'abord rappeler quelques principes clés de la théorie historicoculturelle de l'activité (Cultural Historical Activity Theory: CHAT) et de la théorie de l'activité de troisième génération.

\section{Les trois générations des théories de l'activité historico-culturelles}

Engeström a proposé de distinguer trois générations de recherches dans les théories de l'activité (Engeström, 2000a, 2000b ; 2005). Selon Engeström, la première s'articule autour du concept de médiation de Vygotski. Ce concept est important parce qu'il a surmonté la rupture cartésienne individu/ société, en montrant comment les artefacts culturels médiaient les actions humaines. Ce développement a généré une profonde rupture de la pensée sur le concept d'activité, et depuis, de nombreuses recherches ont employé le concept d'activité médiée. Ceci dit, la recherche est restée limitée dans la mesure où elle s'est centrée largement au niveau de l'individu.

La deuxième génération s'est centrée sur le travail de Leont'ev (voir Engeström 1999; 2000a), qui a le premier mis en avant la nature collective de l'activité. Cette approche conduisait à l'analyse d'interactions complexes entre les individus et leur communauté, et en particulier la manière dont les objectifs collectifs étaient réalisés, par exemple par la division du travail. Elle a ajouté une nouvelle dimension à la théorie, et a permis un bond en avant important puisqu'elle a pu rendre compte de l'action individuelle et collective. En grande partie sous l'influence d'Engeström, qui a introduit à l'Ouest certains concepts issus de la psychologie Russes, la théorie de l'activité a attiré un public international croissant.

Mais selon Engeström (2000a; 2005), une troisième génération de travaux sur la théorie de l'activité doit aujourd'hui être produite pour analyser un système d'activité collectif, médié par l'artefact, orienté vers l'objet au sein de réseaux, et en relation avec d'autres systèmes d'activités. Et pour ce faire, il convient de porter une attention particulière à l'expression d'une diversité ou d'une polyphonie entre différentes perspectives et traditions qui se sont développées au sein des différents systèmes d'activité respectifs. Au sein de cette troisième génération de la théorie historico-culturelle de l'activité, l'accent est mis sur le développement d'outils conceptuels permettant de comprendre 
les « réseaux et perspectives multiples des systèmes d'activité en interaction » (Engeström, 2005, p. 62). Ceci est important au vu de l'interdépendance croissante des différents contextes de travail, comme ceux que l'on trouve dans le contrôle aérien. Comme Engeström le souligne, lorsque les acteurs sont répartis temporellement et spatialement, les actions engagées sont influencées par de nombreux autres facteurs que les communications effectivement réalisées, et ce quelle que soit la situation $(2005$, p. 93).

Engeström utilise trois concepts pour analyser les dimensions qui sont en jeu: le boundary crossing (Engeström, 2001), le « travail en nœud » (Ahonen, Engeström, \& Virkkunen 2000) et la co-configuration (Engeström, 2000a, 2004).

Engeström avait remarqué que le boundary crossing était une catégorie de processus inter-organisationnels et cognitifs vastes, mais peu étudiée. Les chercheurs du Center for Activity Theory and Developmental Work Research ont alors étudié le boundary crossing comme un processus de création de concept, et d'apprentissage collectifs (Engeström 2001; Engeström, Engeström, \& Kerosuo, 2003).

Mais actuellement, deux approches du boundary crossing semblent présentes dans les travaux du Center for Activity Theory and Developmental Work Research. La première approche conceptualise le « boundary crossing » comme un phénomène temporaire, éphémère, désigné sous le terme de « travail en nœud »: La notion de nœud (knot) renvoie à une orchestration de la diversité partiellement improvisée, et rapidement pulsée, de la performance collaborative distribuée, caractérisée par un «mouvement pulsé de nouage, de dénouage et de renouage différents des fils d'une activité... Le centre de l'initiative change moments après moments, dans une séquence de travail en noud» (Engeström, Engeström, \& Vähääho, 1999, p. 346.

Engeström et ses collègues donnent l'exemple d'une collaboration réussie entre le concierge d'un immeuble, d'un voisin et de deux auxiliaires médicaux qui tentent de dialoguer avec une malade mentale. Dans cette conceptualisation, les frontières sont franchies, bien que de façon fugace et éphémère, afin de résoudre un problème temporaire. Pour Engeström et ses collègues, la clé pour comprendre cette nouvelle manière de travailler est de partir du principe qu'il n'existe pas de centre de coordination. Le centre de l'initiative se déplace entre les acteurs et leurs rôles. Cette approche se distingue d'une seconde conceptualisation, celle d'un boundary crossing associé à un apprentissage étendu et une recherche sur le travail développemental qui, si l'on peut dire, est beaucoup plus durable puisqu'il conduit au développement de nouveaux outils. Dans cette conceptualisation, le travail d'intervention conduit au développement de nouveaux outils pour créer de nouvelles formes de travail innovantes. L'exemple donné par Engeström et ses collègues est l'utilisation du change laboratory comme une forme d'intervention développementale.

L'idée de franchissement des frontières et de travail en nœud semble avoir fourni la base pour le développement du concept de co-configuration. S'appuyant sur l'idée développée à l'origine par Victor et Boynton (1998), la co-configuration est, pour Engeström et ses collègues, un nouveau type de travail émergent qui implique un partenariat collaboratif entre des fabricants ou des réseaux de fournisseurs multiples, qui forment des alliances stratégiques avec des clients lorsqu'un ensemble de services ou de produits complexes sont développés (Engeström, 2000a; 2004). Cela dépasse la simple réponse aux besoins du client, car la co-configuration élève la relation société-client à un autre niveau en développant une collaboration interactive dans laquelle "le client devient, d'une certaine manière, le vrai partenaire du fabricant » (Victor, \& Boynton, 1998, p. 199 in Engeström, 2000, p 973). La co-configuration nécessite un « travail en nœud » flexible qui est perçu comme le cœur interactionnel émergeant de la co-configuration, au sein duquel "le centre n'a pas de prise » (Engeström, 2000).

Pour une théorie de l'activité de troisième génération, le défi est alors de développer une compréhension de "la façon dont les actions d'apprentissage finalisées sont entremêlées avec des mouvements horizontaux ou latéraux, et empiètent sur des domaines d'activité complémentaires ou concurrents, 
ce qui est caractéristique de la co-configuration » (Engeström, 2004).

Dans ce papier, je souhaite aborder ce problème. Je souhaite démontrer de quelle manière des pratiques de travail conjointes, qui correspondent à un grand nombre des caractéristiques du « travail en nœud » et qui pourraient donc être considérées comme temporaires et éphémères, surviennent nécessairement à l'intérieur de centres de coordination encore distincts dans des systèmes d'activité, lesquels peuvent avoir « prise », et en réalité doivent « avoir prise ». En démontrant la manière dont des pratiques conjointes sont négociées aux frontières des systèmes d'activité en interaction, je montrerai également, par l'emploi de concepts venus de la théorie organisationnelle, comment ces pratiques avancent selon un mouvement latéral ou horizontal pour résoudre les tensions qui existent lorsque les pratiques de travail conjoint des systèmes d'activité en interaction révèlent des objets contradictoires. La résolution de ces tensions s'inscrit dans des pratiques de travail non pas éphémères et transitoires, mais plus durables, et au sein de systèmes d'activité qui peuvent se développer sur le long terme.

Avant d'expliquer comment le travail entre l'équipage et les contrôleurs aériens peut être analysé de cette façon, il est important de dire quelques mots au sujet de la recherche qui a pu faire émerger les idées exposées ici.

Mais ce papier n'est pas empirique. Au contraire, il développe un cadre théorique qui extrapole à partir de précédentes recherches les implications de cette conceptualisation. Néanmoins, un résumé des données collectées et qui ont abouti à cette formulation est nécessaire pour fournir le contexte.

\section{Recherches antérieures menées sur les systèmes d'activité ATC/équipage}

Les données qui servent à développer mon analyse sont issues de deux études complémentaires. Dans la première, une hypothèse qualitative et explicative a été utilisée, pour laquelle un échantillon de 100 contrôleurs du trafic aérien a été interrogé, souvent plusieurs fois sur une période de trois ans. Les contrôleurs ont été interrogés simultanément dans trois centres de contrôle en Australie, alors que des changements organisationnels importants avaient lieu. Cette étude a permis l'analyse de la façon dont les structures et les cultures organisationnelles permettent et limitent l'apprentissage sur le lieu de travail. Les détails méthodologiques de cette étude et plus largement ses conclusions ont été rapportés ailleurs (Owen, 1999; 2001). La deuxième étude, plus importante, a été menée sur trois ans, et a analysé l'impact des technologies en pleine évolution sur la communication et la collaboration entre les contrôleurs aériens et l'équipage. Au cours de cette étude, un travail d'observation minutieux de 60 heures, portant sur les interactions pilote contrôleur et contrôleur pilote, a été effectué, à des périodes de faible, de moyenne et de forte charge de travail, et sur différents secteurs de contrôle et différents types d'avion. Au cours de cette phase, 83 entretiens, en majorité post-observation, ont été menés (63 entretiens avec des contrôleurs du trafic aérien et 20 avec des pilotes). Au cours de cette période de collecte de données, l'auteur a également exercé le rôle d'animateur-formateur lors de sessions de formation professionnel, ainsi que lors de périodes de changements organisationnels au sein de la société australienne de contrôle du trafic aérien civil.

\section{Conceptualiser le travail de l'équipage et du contrôleur comme des systèmes d'activité en interaction}

Bien que les pilotes puissent faire voler leur appareil tout seuls, ils ne peuvent actuellement pas le faire en coordination avec le reste du trafic. De la même façon, il n'y aurait pas de travail pour un système de contrôle au sol s'il n'y avait pas des compagnies aériennes et des équipages. Pourtant, malgré leur étroite dépendance, le fait est qu'ils ne font pas partie d'un système d'activité unique, mais qu'ils représentent les intérêts qui se chevauchent de deux systèmes d'activité en interaction. Autrement dit, il s'agit du travail conjoint de sujets interdépendants agissants en interaction, et c'est l'exercice professionnel conjoint qui constitue l'unité d'analyse. Dans l'analyse du travail conjoint qu'on va présenter, il apparaît que les éléments de chacun des systèmes d'activité sont évidents, com- 
me le sont les relations entre eux ainsi que les problèmes soulevés à la frontière du travail conjoint, tandis que c'est la trajectoire développementale de l'activité de travail au sein des systèmes d'activité qui crée des tensions entre eux.

\section{Éléments des systèmes d'activité en interaction}

Engeström définit un système d'activité comme un système cohérent du point de vue de l'activité orientée vers des objets, des sujets agissants par l'intermédiaire des artefacts, et organisés collectivement au sein d'une division du travail, des règles qu'ils emploient et de leur communauté (d'intérêts, de pratiques ou de culture) (voir Figure 1)

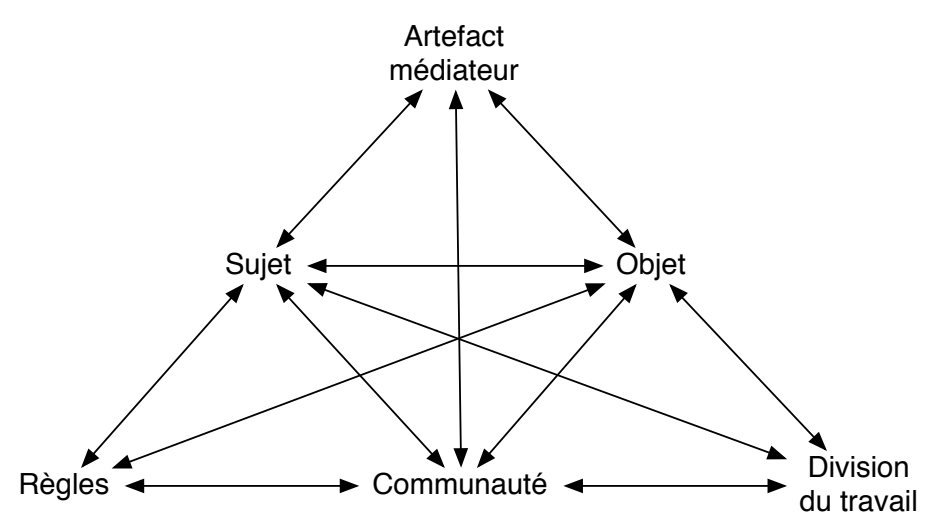

Figure 1: Les composants d'un système d'activité

Figure 1: Components of interacting activity systems

Comme cela est illustré ci-dessous, l'activité orientée-objet d'une compagnie d'aviation représente un système d'activité, dans lequel les sujets agissants sont l'équipage, avec ses artefacts, ses divisions du travail, ses règles et ses communautés; l'autre est le système de contrôle du trafic aérien, représenté par le contrôleur et ses outils, ses règles, sa division du travail et sa communauté. La Figure 2 illustre de quelle manière ces deux systèmes d'activité peuvent être identifiés et leur interconnexion analysée. Au sein de chacun des systèmes d'activité, les sujets agissants (les contrôleurs et les pilotes) ont pour objectif de réussir leur mission orientée - objet. Leurs actions sont médiées par des outils, des règles, des divisions du travail et des communautés de pratiques.

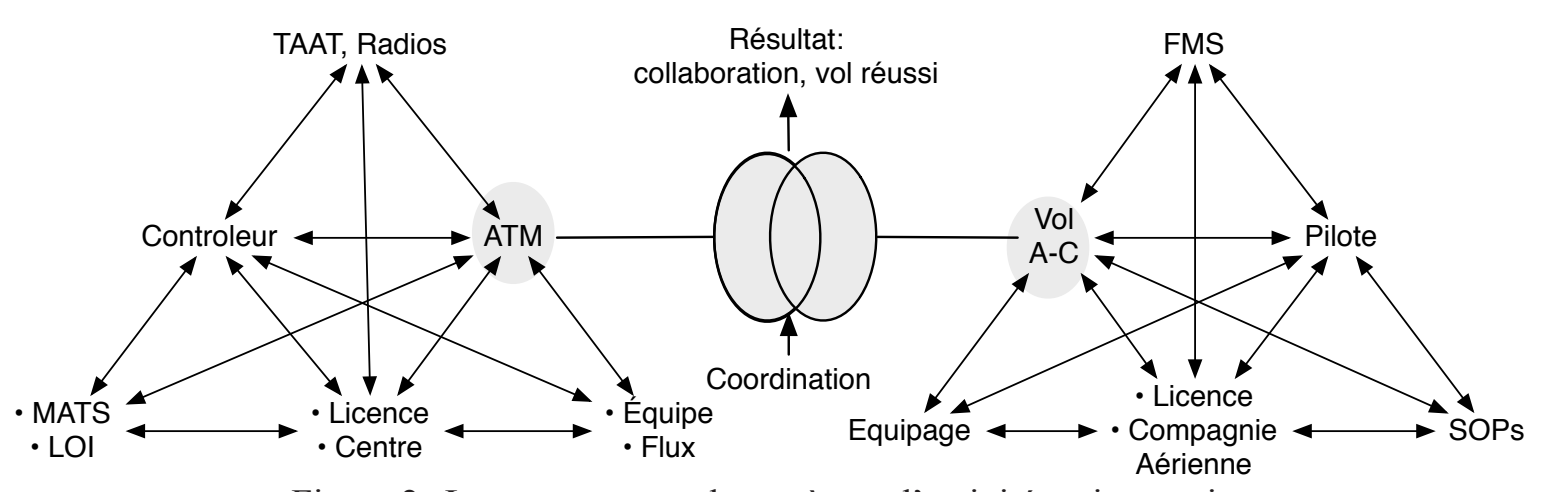

Figure 2: Les composants de systèmes d'activité en interaction

Figure 2: Components of interacting activity systems

Dans la Figure 2, à gauche, le contrôleur (sujet agissant) utilise des outils comme la radio, le radar et des techniques de vol générées par ordinateur (comme celles qui ont été conçues pour le système TAAATS (The Australian Advanced Air Traffic Control System qui permet de gérer le trafic aérien dans des espaces aériens sans radar) afin de gérer l'objet que représente un flux du trafic aérien sécu- 
risé, méthodique et rapide. Le résultat de ces actions est médié par l'utilisation de cette technologie, et est influencé par d'autres facteurs comme les règles (par exemple, les Instructions de fonctionnement locales) qui régissent un espace aérien particulier. Des cultures issues d'un panel de communautés de pratiques auxquelles le contrôleur s'identifie peuvent également être identifiées, et elles influencent également les stratégies que le contrôleur emploie au cours de son travail. Par exemple, elles peuvent inclure l'identification et l'appartenance à des groupes, sur la base de leur affiliation à une partie de la communauté aéronautique (par exemple l'aviation civile ou militaire), l'identification à différents types de secteurs aéronautiques que le contrôleur est habilité à contrôler (par exemple, l'approche/ le départ, les arrivées, la tour de contrôle, les « en route »), etc. La division du travail influence également l'activité de travail. Dans le contrôle aérien, cela inclut par exemple de faire partie d'une équipe qui exploite un flux particulier de la gestion du trafic (par exemple, les « en route », la tour, les arrivées ou l'approche/le départ). Suivant la complexité et le nombre de secteurs pour un espace aérien donné, il peut y avoir trois ou davantage d'équipes mobilisés par exemple.

Un système d'activité similaire guide le travail de l'équipage. Le pilote aux commandes aura pour objet de réaliser une trajectoire sécurisée, méthodique et rapide pour son avion en particulier. Le pilote fera partie d'une équipe composée de deux à quatre personnes, qui utiliseront le système de vol de l'avion, lequel sera composé, dans les cas technologiquement les plus avancés, d'un système de gestion des vols géré par ordinateur. L'activité de travail de l'équipage sera organisée selon une division formelle du travail (le pilote aux commandes, le pilote qui n'est pas aux commandes, l'intervention d'un autre membre du personnel si nécessaire, comme le mécanicien de bord) et des règles (par exemple, des procédures de fonctionnement standard pour cet avion en particulier). Le pilote, lui aussi, fera partie d'une variété de communautés de pratiques qui peuvent inclure des groupes auxquels il s'identifie du fait de son passé (par exemple, l'endroit où il a fait son apprentissage, s'il était dans l'aviation militaire avant de rejoindre l'aviation civile), quel type d'avion il est autorisé à piloter (par exemple, un Boeing 777, un Airbus A320) et pour quel type de compagnie il travaille (par exemple, une compagnie internationale ou une compagnie régionale low-cost).

Durant le déplacement au sein de l'espace aérien, un équipage communiquera avec les contrôleurs qui exploitent parfois entre trois et huit secteurs $\mathrm{ATC}^{2}$ de l'espace aérien au sein de l'unique centre de contrôle australien du trafic aérien. De plus, une partie de ces interactions entre certains contrôleurs et l'équipage ne va durer que trois à cinq minutes (par exemple dans le cas d'un secteur d'approche ou départ d'un aéroport) ou peut durer jusqu'à deux heures (pour un secteur d'en route). Dans ces derniers cas, les actions conjointes des contrôleurs et des pilotes ne surviennent que momentanément.

Dans cette situation, il est intéressant d'examiner tout d'abord la manière dont l'objet du travail est constitué par les sujets agissants respectifs, tandis qu'ils agissent sur les différents systèmes d'activité, et deuxièmement, la manière dont ceci crée (et à son tour est influencé par) des tensions et des contradictions au sein des différents composants du système d'activité dans l'accomplissement de cet objet. Dans l'exemple de la Figure 2, ces deux systèmes d'activité convergent tant qu'ils partagent un objet commun (la gestion conjointe du vol). Il faut noter que les objets du travail ne sont pas toujours bien alignés et qu'ils ne se recouvrent que rarement. Par exemple, lorsque l'objet du travail pour le contrôleur aérien est une gestion sécurisée, méthodique et rapide du flux de la circulation dans un espace aérien donné, l'objet du travail pour l'équipage est le vol sécurisé et rapide de son avion en particulier. Les objets du travail ne se recouvrent que lorsque chacun des sujets agissants sont impliqués dans l'accomplissement conjoint de la gestion du vol.

Toutefois, l'accomplissement conjoint de cet objet mutuellement constitué est parfois soumis à des tensions, du fait de l'influence des exigences au sein de chacun des systèmes d'activité respectifs. Par exemple, au cours d'un vol, il est probable que le pilote veuille s'assurer de l'optimisation de sa consommation de carburant (certains transporteurs aériens distribuent en fin de mois une prime au pilote s'il a économisé du carburant). Le pilote aura également intérêt à s'assurer que l'avion vole selon sa courbe de performance optimale et que les passagers ne sont pas gênés par un retard ou

2. Un ATC est un volume donné de l'espace aérien (NdT). 
des turbulences (faire autrement ne serait pas professionnel). D'un autre côté, l'intérêt principal des contrôleurs du trafic aérien est de gérer les demandes simultanées qui leur échoient afin d'optimiser le flux du trafic dans un espace aérien donné. Cela signifie par exemple, qu'un contrôleur aérien, pour gérer le flux de la circulation aérienne, peut diriger un pilote d'une manière qui est à opposer à ce qui est souhaité par ce dernier (comme placer l'avion dans un ordre d'atterrissage qui va reporter l'heure d'arrivée prévue de l'avion), il va sans doute générer de la tension qui produira parfois une contestation de la part du pilote. ${ }^{3 .}$ Ces stratégies ont été rapportées par Besco (2000), par exemple lorsque les pilotes ont parfois arrangé la vérité au sujet de leur position et de l'estimation de leur heure d'arrivée, afin de s'assurer d'une place avantageuse dans la file d'attente pour l'atterrissage.

Ces développements théoriques laissent entrevoir de grandes possibilités d'exploration d'un nouveau territoire de travail conjoint situé aux frontières des systèmes d'activité. Engeström (2005) propose ce qu'il a défini comme un concept provisoire, et qu'il appelle « une inter-organisation orientée objet ». Pourtant, il est nécessaire de l'étoffer encore afin de fournir un tableau complet d'une pratique de travail, telle qu'elle survient à l'intersection de différents systèmes d'activité en interaction. Ce papier vise à contribuer à ce développement.

L'ennui est que l'analyse se porte encore au sein des systèmes d'activité, et accorde une attention limitée au travail négocié entre une organisation ou un domaine de travail et une autre, malgré le nombre croissant d'interconnexions entre l'activité de travail et les systèmes. Engeström (2000a) identifie cette faiblesse lorsqu'il note que, malgré des contextes et des formes de travail organisationnel émergents, le boundary crossing reste une catégorie des processus cognitifs vaste, mais très peu étudiée.

\section{Comprendre les pratiques de travail à la frontière de différents systèmes d'activité}

Comment cette analyse pourrait-elle aider à comprendre le travail conjoint des contrôleurs et de l'équipage, et ce qui permet ou limite une réalisation collaborative réussie? Dans le cas présenté plus haut, on peut identifier deux systèmes d'activité « frontiérisés » par les usages de leurs outils respectifs, de leurs règles, des divisions du travail et des objets. Le travail des sujets agissants respectif au sein de ces systèmes d'activité repose toutefois sur les interactions qui se jouent avec l'autre. Les moyens qui servent à exécuter ce travail existent par l'intermédiaire d'un « objet frontière » ou d'une interface commune (Nardi, 1996), comme la communication par l'intermédiaire des technologies employées. Pourtant, comme nous allons le voir, il existe également des frontières entre les systèmes d'activité qui définissent leur démarcation. Ces frontières peuvent ou non être prises en charge par des interfaces adaptées susceptibles de les mettre en relation.

Dans ce papier, j'analyse les frontières telles qu'elles surviennent entre les sujets agissants qui réalisent par médiation un travail conjoint, grâce à aux éléments suivants :

- L'intersection de leurs artefacts ou technologies - frontières d'interopérabilité respectives -;

- L'application de leurs règles - frontières de responsabilité -;

- Les interactions de leurs divisions du travail respectives - frontières de responsabilité du rôle -;

- Les ressemblances et les différences de leurs communautés culturelles professionnelles frontières de l'identité sociale -.

D'autres frontières peuvent également être identifiées. Puisque les caractéristiques citées représentent les attributs principaux mis en avant dans les systèmes de théorie de l'activité, elles serviront de point de départ à cette analyse.

L'aperçu des deux systèmes d'activité a permis de mettre en évidence la manière dont les deux systè-

3. Notez que cela ne signifie pas qu'un pilote enfreindra délibérément cet ordre, simplement que cela pourra générer des discussions ou des contestations. 
mes peuvent être représentés dans une pratique conjointe et comment les objets situés à l'intersection des sujets agissants peuvent empiéter les uns sur les autres, tout en n'étant pas exactement identiques. Ce papier va maintenant analyser ce que la négociation des frontières de ces systèmes d'activité révèle du développement d'une pratique conjointe.

\section{Les frontières de l'interopérabilité}

Selon la théorie de l'activité, les outils sont des artefacts qui opèrent une médiation entre le sujet et l'objet de cette activité (voir Figure 1). Engeström (2005) met en évidence la façon dont ces outils peuvent être à la fois cognitifs [par exemple, un modèle mental que le sujet utilise, (voir par exemple Engeström, Engeström, \& Kärkkäinen, 1995) pour une analyse] et technologiques. Pour une analyse des systèmes d'activité en intersection, il est utile d'étudier la façon dont les systèmes technologiques sont interconnectés et les implications que cela génère pour les outils cognitifs employés par les différents sujets agissants. Comme nous l'avons dit plus haut, il a été souligné (Kerns, 1999) que les technologies utilisées dans l'aviation ont été développées au sein de chaque système d'activité, de l'équipage et du contrôle au sol, et que par conséquent, elles ne sont pas vraiment faites pour être utilisées en coordination des différentes organisations dans l'accomplissement d'un but commun. Par exemple, le contrôleur travaille avec une vue en plan bidimensionnelle du trafic, qui est très bien adaptée aux procédures de séparations radar et à la représentation des solutions vectorielles appliquées aux problèmes d'espace et de séparation. En revanche, les systèmes de gestion des vols utilisés par les pilotes de ligne fournissent une courbe qui indique le profil vertical à toutes les étapes du vol, ceci afin de gérer la consommation de carburant et de respecter les durées de vol. En conséquence, et en accord avec Kerns (1999), lorsqu'un contrôleur donne l'instruction par radio de diriger l'avion hors route (comme ils le font souvent), cette instruction met une pression cognitive sur le pilote qui tâche d'exécuter et de conserver la trajectoire de l'avion qui lui est imposée avec ses outils, mais qui serait moindre s'il utilisait les outils des contrôleurs.

En termes de trajectoire développementale du travail, c'est le radiotéléphone qui est actuellement l'interface la plus généralement partagée entre le poste de contrôle et le poste de pilotage. Jusqu'à récemment, presque toutes les informations et les autorisations issues du trafic aérien étaient transmises par radio. Dans l'aviation, le style de communication a été conçu pour garantir que les communications orales soient transmises de manière efficace et rapide sur des canaux souvent fréquemment utilisés par les opérateurs. Cependant, l'augmentation du trafic a eu pour résultat de surcharger ce médium. Ceci parce que seule une personne peut parler à la fois et dans les environnements de grande activité, cette concurrence entraîne du délai dans le relais des messages, ou la coupure de la ligne. De plus, dans des secteurs océaniques, il est souvent difficile d'entendre ou d'avoir accès à des radios à très haute fréquence. L'outil innovant qui a été développé est la liaison C/P-DLC (Controller Pilot Data Link Communication). La liaison C/P-DLC comporte un certain nombre d'avantages et d'inconvénients qui sortent du cadre de cet article (voir Kerns, 1999). Les tensions qui résultent de la mise en place de ce nouvel outil sont dues aux conséquences de la perte des informations de lignes partagées, auxquelles les pilotes avaient l'habitude d'avoir accès lorsqu'ils écoutaient la radio (par exemple, le dialogue entre les autres pilotes et les ATC compétents sur la même fréquence). Une technologie apparentée a été développée pour permettre une conscience de la situation globale dans la cabine de pilotage: la technologie CDTI (Cockpit Display of Traffic Information). Cet outil permet aux pilotes dont le poste est équipé en conséquence de détecter, par l'intermédiaire d'un écran, d'autres avions situés dans leur espace aérien. La technologie CDTI fournit ainsi aux pilotes l'accès aux mêmes informations que celles dont disposent les contrôleurs au sol. Les possibilités offertes par ces technologies émergentes tendent à modifier la division du travail entre les contrôleurs et les pilotes, et pose des questions sur leurs rôles et leurs responsabilités respectives.

Il est important de comprendre que ces nouvelles créations technologiques ne remplacent pas les anciens systèmes, mais qu'elles s'y ajoutent. Cela signifie qu'il y a une augmentation de la diversité 
des avions qui utilisent des technologies différentes, avec un nombre croissant d'interfaces d'interopérabilité avec lesquelles le contrôleur doit composer.

Les questions clés pour des recherches futures pour l'analyse et l'intervention sur les systèmes sont les suivantes:

- Où se situe la frontière entre les artefacts technologiquement intégrés et quelles sont les implications de la charge cognitive des sujets agissants qui les utilisent?

- Est-ce que le nombre de frontières à négocier augmente ou décroît, et quel est le nombre qu'un sujet agissant est raisonnablement capable de gérer sans dégradation de sa performance?

Les spécialistes de la théorie de l'activité ne s'étonneront pas que ces changements aient des conséquences sur d'autres aspects du système d'activité.

\section{Les frontières de responsabilité}

Selon les termes de la théorie de l'activité, les règles renvoient « aux règlements, normes et conventions explicites et implicites qui limitent les actions et les interactions au sein du système d'activité » (Engeström, 1999, p. 79). Dans les organisations extrêmement formelles, telles que l'aviation, la discrétion et l'autonomie de leurs membres sont plus limitées que dans les organisations moins formelles, où l'exercice du choix est plus libre. De cette façon, comme Flach (1999) le remarque, les règles opèrent toujours des compromis entre la liberté, l'autonomie et entre les règles elles-mêmes.

Le terme de responsabilité est utilisé ici dans deux sens. Lorsqu'une personne applique (ou contourne) des règles, elle est « responsable» de ses pratiques d'une certaine manière (Suchman, 1993; 2000). Deuxièmement, les dirigeants tiennent une personne pour « responsable » en termes de sanctions ou de soutien vis-à-vis de ses actions (McCarthy, Healey, Wright, \& Harrison, 1997). Pour analyser comment les sujets agissants développent des pratiques qui impliquent la négociation de règles à l'intersection entre différents systèmes de règles, il est utile de s'appuyer sur des cadres qui permettront d'examiner l'organisation des règles et d'analyser ces problématiques de responsabilité (personnelles autant qu'organisationnelles). En étudiant l'application des règles dans les organisations à haut risque, McCarthy et al. (1997) identifie un ensemble de dimensions qui dirigent l'attention vers les relations qu'entretiennent une responsabilité formelle et une activité de travail dans des contextes différents. Celles-ci ont été adaptées ici et comprennent les caractéristiques suivantes:

1.- Explicite - implicite: indique jusqu'à quel point l'application des règles et des procédures développées est présentée sous une forme qui est disponible pour une inspection externe (explicite) ou non (implicite).

2.- Globale - locale: indique jusqu'à quel point les règles et les procédures sont structurées globalement ou localement. Une règle est structurée globalement lorsque des personnes autres que celles qui sont impliquées dans l'activité de travail l'imposent. Qu'une règle soit globalement structurée dépend également de l'étendue de la responsabilité des personnes impliquées.

3.- Stable - Fluctuante: indique l'étendue de la flexibilité impliquée dans une situation. C'est-àdire dans quelle mesure l'application des règles et des procédures est fixe ou peut changer en fonction des situations.

4.- Dépendante-indépendante: indique dans quelle mesure les règles et les procédures peuvent être séparées les unes des autres, ou dépendantes les unes des autres.

Dans les environnements de travail complexes, les règles et les procédures peuvent être établies sur la base de leur position dans chacune de ces dimensions. Au vu de l'importance que donne la théorie de l'activité aux processus développementaux au travail, il est aussi utile d'analyser dans quelle mesure ces dimensions sont impactées au gré des changements dans les systèmes de travail. Si l'on étudie le travail conjoint situé à l'intersection des systèmes d'activité, deux questions sont intéres- 
santes. Premièrement, au vu de la trajectoire historique d'un système d'activité, de quelle manière ces dimensions de règles (et l'application de ces règles et procédures) changent-elles en relation avec l'accomplissement des objets communs? Et deuxièmement, quelles en sont les implications sur un travail situé à la frontière des systèmes d'activité connectés? Par exemple, dans l'aviation australienne, les avancées qui visent le développement de nouveaux artefacts comme la liaison C/P-DLC sont autorisées en partie du fait de leur précision à localiser l'emplacement spatial d'un avion donné (de par leur dépendance envers d'autres outils comme les satellites). Ces changements peuvent conduire à rendre explicites certaines règles qui étaient auparavant en grande partie implicites, et en rendre d'autres qui étaient stables à un moment donné, plutôt fluctuantes dans les circonstances à venir.

Donnons un autre exemple. L'un des objectifs clés d'une compagnie aérienne est de satisfaire des clients qui penseront qu'ils auront fait une bonne affaire avec une compagnie aérienne qui fait grand cas de leurs intérêts. En fonction de cet objet désiré, les pilotes quittent fréquemment la trajectoire donnée par les contrôleurs (lorsqu'ils estiment qu'il n'y a pas de risque) pour fournir une vue aérienne des sites touristiques (par exemple, un survol du massif d'Uluru lors d'un atterrissage à Alice Springs). Des tensions émergent actuellement parce que les avions équipés du système de traçage ETOPS (par satellite) effectuent ce type de déviations, visibles désormais sur la console TAAATS comme se trouvant hors route (alors que les avions non équipés de cette technologie n'apparaissent pas). Ainsi, le contournement de cette règle sera transparent pour le système d'activité de contrôle aérien si les avions sont équipés de certains outils technologiques, mais opaque pour les autres avions. De même, lorsqu'un contrôleur a l'opportunité de partager l'objet du travail avec le pilote, par exemple d'aider ce pilote à maintenir une trajectoire optimale de l'aéronef, le contrôleur peut collaborer avec le pilote (lorsque c'est possible) pour contourner les règles, en invoquant un schéma de communication qui l'y autorise. Par exemple, en réponse à la requête d'un pilote demandant à descendre, un contrôleur pourra dire « afin de faciliter la descente, je vous demande de vous positionner 60 miles plein Ouest de... », ce à quoi un pilote répondra que l'avion vient d'atteindre cette position. Les contrôleurs font « ce qu'il faut » pour qu'un vol soit réussi, même s'il faut fournir un service qui correspond à un désir du pilote de contourner les règles.

Toutefois, il existe également des tensions entre pilotes et contrôleurs au regard de l'application des règles de séparation de l'espace aérien dans la gestion du trafic aérien. En réponse à la question «Qu'aimeriez-vous que les pilotes connaissent au sujet de votre travail de contrôleur? », l'une des personnes interrogées a répondu qu'il préférait identifier ce qu'il ne voulait pas que les pilotes sachent, à savoir le calcul des normes de séparation latérale appliquées aux avions sur des trajectoires convergentes. Le contrôleur ne voulait pas que cette information sur l'application d'une règle du contrôle du trafic aérien soit connue de la communauté des pilotes, car il pensait que pour obtenir ce qu'ils voulaient, certains pilotes essayeraient d'anticiper la règle de séparation de l'espace aérien et de fournir l'information dont ils pensaient que le contrôleur avait besoin.

Les questions clés pour une étude plus poussée sont les suivantes:

- Dans quelle mesure les changements dans l'application des règles et des procédures sont influencées par l'évolution des technologies?

- Quelle est la nature (explicite/implicite, globale/locale, stable/fluctuante, dépendante/ interdépendante) des règles appliquées à un travail conjoint entre différents systèmes d'activité, comment ces dernières changent-elles et qu'est-ce que cette analyse révèle de comment les sujets agissants développent des pratiques de travail conjoint?

- Dans quelle mesure les négociations entre les sujets agissants se modifient, selon les changements qui affectent les autres systèmes d'activité, comme l'évolution technologique?

Cette brève analyse souligne le fait que les recherches sur la troisième génération des théories de l'activité doivent analyser la nature des règles et de leurs dimensions, ainsi que les conséquences qu'ont les changements survenant dans ces dimensions sur les frontières entre les systèmes d'activité. 


\section{Les frontières de la responsabilité du rôle}

À l'autre extrémité du cadre du système d'activité se trouve la division du travail, que nous allons discuter à cause du lien étroit qu'elle entretient avec les règles. La division du travail renvoie à la fois à la division horizontale des tâches, et à la division verticale du pouvoir et du statut (Engeström, 2005). En termes de théorie des organisations, ces divisions du travail ont un rapport avec les problématiques de complexité, de centralisation et d'exercice du pouvoir dans les organisations. Lorsqu'elles gèrent la complexité, les organisations utilisent différentes stratégies en termes de différentiation et d'intégration (Jones, 1995) de manière à diviser le travail et à le coordonner. La différentiation est le processus par lequel les personnes et les ressources sont affectées à des rôles, des tâches et des fonctions, comme cela est mis en évidence dans la division du travail des organisations, tandis que l'intégration renvoie aux stratégies utilisées pour coordonner ces tâches, ces rôles et ces fonctions (Jones, 1995). Engeström (2005) fait la distinction entre la différentiation qu'on trouve dans une hiérarchie organisationnelle (différentiation verticale) et celle qu'on trouve dans la manière dont les tâches et les rôles sont organisés en sous-unités, fonctions ou départements (différentiation horizontale).

Les commentateurs d'une approche organisationnelle (par exemple, Jones, 1995) prétendent que le défi pour les organisations est de trouver un équilibre entre la différentiation et l'intégration. Par exemple, les innovations technologiques présentées au début de ce papier ont eu pour résultat le développement d'une tension sur la question de la division des rôles et des responsabilités entre l'équipage et les contrôleurs au sujet de la gestion du plan de vol. Certaines sections de l'industrie aéronautique essaient d'imposer une réévaluation des responsabilités des fonctions à ce sujet (la gestion des plans de vol). Sur la table des négociations de différents forums internationaux, on trouve la notion de « vol libre », par laquelle les pilotes changent leur propre trajectoire de vol et effectuent la « séparation » de leur propre appareil dans certains espaces aériens, dans la mesure où ils entrent dans ces espaces aériens et les quittent par certaines « portes » virtuelles. Cette division du rôle et de la responsabilité reviendrait à donner un rôle plus important à l'équipage dans la mesure où il pourrait se séparer du reste du trafic.

Une autre division potentielle du travail entre les deux systèmes d'activité est la possibilité pour le contrôleur de mettre en place la séquence d'atterrissage, à charge pour l'équipage de gérer la distance de séparation entre son appareil et les autres. Ceci a conduit certains, dans l'industrie (Kreifedlt, cité dans Kerns, 1999), à conclure que les pilotes peuvent maintenir les normes de séparation dans l'espace aérien mieux que les contrôleurs.

Les questions clés, qui présentent un intérêt dans le cadre de ce papier, sont les suivantes:

- Quelles sont les conséquences des changements d'artefacts sur la division du travail au sein de différents systèmes d'activité, dont l'objectif est d'accomplir un travail conjoint entre différents systèmes d'activité ?

- Quels sont les points de contestation qui surgissent sur la manière dont la division du travail est organisée dans le travail conjoint, et qu'est-ce que cela révèle de la conceptualisation des objets partagés?

La problématique ici est, en partie, le statut perçu et le pouvoir des groupes respectifs qui sont à leur tour influencés par l'identité sociale des groupes.

\section{Les frontières de l'identité sociale}

Au cœur du concept de « communauté » se trouve l'idée que les individus partagent ce qu'ils savent, de manière formelle ou informelle, parce qu'ils appartiennent et s'identifient à un groupe particulier. Engeström (1999) utilise le mot « communauté » pour décrire des individualités multiples et/ou des sous-groupes qui partagent le même objet d'ordre général. Blackler, Crump et McDonald (1997) 
parlent de « communauté d'activité » dans laquelle un savoir-faire et un apprentissage sont reconnus comme un phénomène collectif. Dans ce papier, les groupes possèdent par définition un sens de la communauté lorsqu'ils partagent une identité au sein d'une infrastructure cognitive et technologique commune.

Pour les communautés d'activité (ou, comme certains théoriciens préfèrent le dire, les communautés de pratiques) l'accent est mis sur l'interaction, à la fois formelle et informelle, et sur la façon dont la mémoire collective se construit sur le long terme. Cette précision nous aide également à comprendre la façon qu'ont les groupes d'individus interdépendants d'apporter le contexte dans lequel les participants construisent des identités individuelles et sociales, et la façon dont le contexte social permet à ces identités d'être partagées (Brown, \& Duguid, 2001).

Lorsque des individus se définissent comme faisant partie d'un groupe, ils justifient leur comportement en termes de normes de groupe et développent une identité de groupe par leurs interactions avec les autres personnes du groupe et par les réponses des autres à un comportement de groupe (Fine, 1996). Selon les théories de la cognition sociale (Augoustinos, \& Walker, 1995), les croyances et les valeurs arbitrent l'interprétation de l'expérience de travail et à leur tour, façonnent les éléments considérés comme importants pour l'appartenance intra-groupe. Elles deviennent des attributs que l'on souhaite présenter à ses pairs et deviennent ainsi une partie de l'expression de soi vis-à-vis du groupe. Adhérer à certains aspects particuliers de ces croyances et de ces valeurs revient à définir le groupe et ainsi, une partie de son identité sociale (Augoustinos, \& Walker, 1995) en ce que «l'identité sociale représente cette partie de l'image de soi individuelle, qui provient de leur connaissance, de leur appartenance à un (ou plusieurs) groupe social, à laquelle se rajoutent la valeur et la signification émotionnelle de cette appartenance » (p. 98).

Il y a parfois des tensions entre différentes communautés de pratique au sein de systèmes d'activité en interaction, en partie du fait des identités sociales respectives des sujets agissants. Par exemple, les contrôleurs comme les pilotes appartiennent à une élite professionnelle soumise à la complexité du travail, ainsi qu'aux pratiques rigoureuses de sélection et d'exigence de formation qu'implique ce travail. Cependant, il existe une compétition entre les communautés parce que les pilotes ont un statut et une rémunération supérieurs à ceux de leurs homologues au sol (Besco, 2000). Une rivalité professionnelle entre les deux groupes a été observée par Besco (2000, p 87) qui mentionne la contrariété des contrôleurs au prétexte que les pilotes « obtiennent à la fois l'argent et les jolies filles ». Cette rivalité entraîne des conséquences sur les actions des deux parties. Selon Besco (2000), les pilotes peuvent être contrariés par un contrôleur lorsqu'une autorisation de décollage ou d'atterrissage n'a pas donné la priorité à tel pilote, plutôt qu'à tel autre. Dans ces circonstances, comme nous l'avons vu, des pilotes ont déjà altéré « la vérité » sur le temps de vol estimé pour bénéficier d'une priorité supérieure (Besco, 2000). Grâce aux développements des technologies en intersection avec les systèmes d'activité, ces tentatives sont dévoilées. Besco (2000) observe également que parfois les contrôleurs du trafic aérien ne tolèrent aucune erreur des pilotes. En retour, certains pilotes ne sont pas disposés à révéler une erreur ou leur confusion. Cela peut conduire à des formulations peu précises ou à une absence de partage de l'information. Comme le rôle de chaque partie change du fait des développements technologiques (par exemple, le mouvement vers le « vol libre » où le pilote a son mot à dire pour la définition de sa propre trajectoire), les tensions s'accumulent. La plaisanterie "vous être à terre parce que je suis là-haut, ou bien, je suis là-haut parce que vous êtes à terre? " est parfois évoquée par les pilotes, pour remettre les contrôleurs à leur place. Toutefois, historiquement, ces différences ne sont pas si évidentes.

L'un des principes centraux de la théorie de l'activité est que l'existence de contradictions dans l'activité de travail peut être révélée par l'étude de la trajectoire historique du travail en question. L'histoire de la pratique du travail dans l'aviation australienne révèle que les communautés de pratiques des pilotes et des contrôleurs se chevauchaient davantage de par le passé. Ces chevauchements étaient facilités par la nécessité et les opportunités d'interagir physiquement. De fait, au début de l'aviation civile en Australie après la deuxième Guerre mondiale, les contrôleurs de trafic aérien civil 
étaient recrutés parmi les ex-pilotes militaires. Pendant deux décennies, le recrutement des contrôleurs aériens s'effectuait sur une population professionnelle issue de l'aviation, ce qui signifiait que la personne avait soit un brevet de pilotage, soit un passé dans l'armée de l'air, ou bien que son père avait servi dans l'aviation. ${ }^{4}$ Lorsque la main-d'œuvre militaire fut épuisée dans les années 60 , les pratiques de recrutement changèrent et pour la première fois, les contrôleurs furent recrutés dans le civil.

En Australie, jusqu'au début des années 90, de nombreux aéroports et aérogares régionales éloignés employaient des contrôleurs du trafic aérien qui débutaient leur carrière à la campagne, pour gagner de l'expérience, avant d'être embauchés dans des secteurs d'espace aérien davantage chargés et plus rapides, reliant la majorité des capitales. Dans ces endroits reculés du pays, les contrôleurs pilotaient eux-mêmes des avions légers, et même lorsque ce n'était pas le cas, ils avaient fréquemment l'opportunité de résoudre un différent d'opinion avec un pilote au bar de l'aéro-club local où, selon des anciens interrogés, plus d'un incident aérien avait été discuté et résolu.

Historiquement, c'est également dans ces environnements éloignés que de nombreux pilotes apprenaient leur métier et gagnaient de l'expérience. Désormais, la professionnalisation de ces deux métiers nécessite une formation rationalisée d'un niveau universitaire, ce qui laisse peu d'opportunité d'apprentissage informel partagé dans un environnement de formation ${ }^{5}$. Les impératifs économiques ont aussi entraîné une rationalisation des pratiques de formation, notamment le retrait des composants de type « c'est bon à savoir » des programmes de formation, comme les stages dans les compagnies aériennes (où les contrôleurs effectuaient leur stage à bord, au poste de pilotage). Ces changements conduisent à une spécialisation accrue et à un déclin des opportunités d'apprendre de manière informelle certains aspects du système aéronautique et sur son rôle propre au sein de ce système. Dans le passé, c'était le rôle d'une communauté de pratiques partagées. Parce que des changements impactent de nombreux autres éléments à l'œuvre au sein de ce système d'activité, l'accès à ce répertoire partagé d'expériences est en train de se réduire. Dans ces circonstances, l'évolution des artefacts technologiques, les changements structurels qu'imposent les nouvelles règles de recrutement et d'engagement dans le travail (comme le dépôt automatique des plans de vol), la modification profonde de la division du travail pour aller vers une centralisation des services en deux centres, ainsi que le passage à une structure d'équipe, tout cela affecte le stock des ressources partagées qui permet de réussir un travail conjoint dans cet espace virtuel. Cette analyse soulève les questions suivantes, à prendre en compte lors d'une intervention dans ces environnements de travail:

- Quelle mémoire collective a été développée au cours du temps dans les communautés de pratiques respectives?

- Quel rôle a tenu de manière informelle la mémoire collective, au sein de la communauté de pratiques, dans l'apprentissage et dans le désapprentissage (nécessaire pour faciliter le changement dans les organisations)?

- De quelle manière les changements survenus dans le système d'activité ont-ils impacté la trajectoire historique de l'interaction au sein des communautés de pratiques respectives et entre elles?

Ces types de questions sont apparentés aux questions soulevées par l'analyse des autres nœuds (knots), situés au sein des systèmes d'activité. Une analyse de la totalité du système d'activité, qui s'appuie sur une étude des nœuds, devrait fournir les moyens d'une analyse cohérente.

\section{Conclusion}

Ce papier a tenté d'apporter une contribution à la troisième génération de la théorie de l'activité.

4. Il est intéressant de noter que l'idée qu'être un bon contrôleur est inné et ne s'apprend pas, perdure pour certains contrôleurs, ce qui a des conséquences évidentes sur leur formation. Toutefois, ceci sort du cadre de ce papier.

5. Il est intéressant de noter que dans certains pays, une formation conjointe (des pilotes et des contrôleurs) est actuellement réalisée. 
Il a illustré la manière dont le travail conjoint, situé aux frontières de deux systèmes d'activité en interaction, permet et limite les technologies, les règles, les divisions du travail et l'identité sociale en interaction. Ce papier tente d'améliorer la compréhension de la théorie de l'activité de troisième génération en s'appuyant sur des concepts employés dans les théories des organisations au sujet de l'utilisation des règles et de l'organisation du travail pour répondre aux problématiques liées à la complexité du travail, comme la différentiation et l'intégration du rôle, ou l'identité et la culture professionnelles. Ce faisant, il a montré comment les dimensions des systèmes d'activité en interaction sont impliquées dans un travail conjoint situé aux frontières de ces systèmes d'activité.

Lorsqu'ils sont engagés dans une inter-organisation orientée objet, les sujets agissants développent au cours du temps des pratiques délimitées par des tensions et des contradictions qui émergent historiquement dans un système d'activité en interaction. Par exemple, l'analyse de la façon dont une inter-organisation orientée objet est médiée par les outils utilisés par chacun des systèmes d'activité respectifs démontre que la pratique du travail conjoint est rendue difficile par le développement du système qui a créé les technologies destinées à fonctionner au sein du système d'activité, plutôt que d'améliorer l'interopérabilité entre eux. L'un des exemples de résolution de ces difficultés est la création de plates-formes d'intégration de systèmes grâce à l'introduction de nouveaux outils comme les liaisons C/P-DLC et CDTI, qui fournissent aux équipages une amélioration d'ordre cognitif, par l'intégration dans le poste de pilotage d'outils qui fournissent les informations dont disposent les contrôleurs. Cependant, ces changements créent des contradictions secondaires médiées par les frontières de l'identité sociale (la communauté), qui à leur tour entrainent des changements aux frontières de la responsabilité du rôle (division du travail). Ces changements impliquent également d'autres contradictions dans les pratiques de travail existantes, en modifiant les limites du comportement gouverné par les règles.

Recadrer les pratiques de travail entre équipage et contrôleur, à la frontière de deux systèmes d'activité en interaction, améliore également la compréhension du travail dans ce domaine et en particulier de ce qui permet ou limite la réussite de l'accomplissement conjoint des pratiques de travail. Améliorer l'interdépendance du travail dans l'industrie aéronautique est depuis longtemps une préoccupation, mais jusqu'à présent, une grande partie de l'attention portée à cette problématique s'est focalisée sur les pratiques ou l'interopérabilité de communication au sein des systèmes technologiques. Ce papier définit une série de frontières qui représentent des sources de tension développementale et de contradictions systémiques dans les systèmes d'activité en interaction.

Analyser le travail collaboratif du contrôle du trafic aérien et de l'équipage comme une représentation d'un travail conjoint situé aux frontières de systèmes d'activité en interaction apporte une explication théorique plus satisfaisante, car elle met davantage l'accent sur le caractère multicouche que ne le fait la littérature sur les facteurs humains et l'aviation. Cette analyse permet de plus grandes opportunités pour le professionnel qui souhaite intervenir au sein de systèmes complexes en plein changements, car elle insiste sur la manière dont on peut résoudre les problèmes situés à la frontière d'une pratique.

Cette analyse soulève de nouvelles questions, qui sont destinées à aider les personnes impliquées dans la conception des interventions dans ces environnements de travail. Ce sont des questions qui aident à poser un diagnostic, et que les professionnels et les spécialistes qui souhaitent améliorer la perspective d'une pratique conjointe seront amenés à se poser. Ces questions visent à fournir un véhicule pour améliorer le travail conjoint situé aux frontières de différents systèmes d'activité en interaction, une zone qui, au vu de l'augmentation de l'interdépendance dans le travail, est susceptible d'être l'objet d'un intérêt croissant. Grâce à cette analyse, ce papier espère contribuer à notre compréhension collective de ces problématiques au sein de la recherche sur la théorie de l'activité de troisième génération. 


\section{RÉFÉRENCES}

Ahonen, H., Engeström, Y., \& Virkkunen. (2000). Knowledge management - the second generation: creating competencies within and between work communities in the competence laboratory. In Y. Malhotra (Ed.) Knowledge Management in Virtual Organizations (pp. 282-305). Harrisburg, PA:IGI Publishing.

Augoustinos, M., \& Walker, I. (1995). Social cognition. London: Sage.

Baker, D.P., \& Salas, E. (1992). Principles for measuring teamwork skills. Human Factors, 34 (4), 469-475.

Besco, R. (2000). Pilot/controller teamwork: an awkward alliance. Business and Commercial Aviation, Sept, $82-88$.

Blackler, F., Crump, N., \& McDonald, S. (1997). Crossing boundaries: some problems of achieving expansive learning in a high technology organization. Paper presented at the EIASM Conference on Organizing in a multi-voiced world. Leuven: Belgium.

Boreham N., Samurçay, R., \& Fischer, M. (2002) Work process knowledge in technological and organizational development. In N. Boreham, R. Samurçay, \& M. Fischer (Eds.), Work Process Knowledge (pp. 1-14). London: Routledge.

Casey, C. (1999). The changing contexts of work. Understanding learning at work. In D. Boud, \& J. Garrick (Eds). The changing contexts of work (pp. 15-28). London: Routledge.

Cushing, S. (1995). Pilot-air traffic control communications: it's not (only) what you say; it's how you say it. Flight Safety Foundation. Flight Safety Digest, 1-10.

Dekker, S.W.A. (2000). Controllers or exception managers? To intervene or not to intervene - the central problem in future air traffic control. Flight Deck, 33, 48-51.

Engeström, Y. (1999). Expansive visibilization at work: an activity theoretical perspective. Computer Supported Co-operative Work, 8, 63-93.

Engeström, Y. (2000a). From individual action to collective activity and back: developmental work research as an interventionist methodology. In P. Luff, J. Hindmarch, \& C. Heath (Eds.), Workplace studies: Recovering work practice and informing system design (pp. 150-166). Cambridge: Cambridge University Press.

Engeström, Y. (2000b). Activity theory as a framework for analyzing and redesigning work. Ergonomics 43(7): $960-974$

Engeström, Y. (2001) Expansive learning at work: toward an activty theoretical reconceptualization. Journal of Education and Work, 14(1), 133-156.

Engeström, Y. (2004). New forms of learning in co-configuration work. Journal of Workplace Learning, 16, $11-21$

Engeström, Y. (2005). Developmental work research: expanding activity theory into practice. Berlin: Lehmanns Media.

Engeström, Y. (2006) From well-bounded ethnographies to intervening in Mycorrhizae activities. Organization Studies, 27, 1783- 1793.

Engeström, Y., Engeström, R., \& Karkkainen, M. (1995). Polycontextuality and boundary crossing in expert cognition: learning and problem solving in complex work activities. Learning and Instruction, 5, 319336.

Engeström, Y., Engeström, R, \& Kerosuo, H. (2003). The discursive construction of collaborative care. Applied Linguistics, 24, 286-315.

Engeström, Y., Engeström, R., \& Vähääho, T. (1999). When the center does not hold: The importance of knotworking. In S. Chaiklin, M. Hedegaard, \& U.J. Jensen (Eds.), Activity theory and social practice (pp. 345-374). Aarhus: Aarhus University Press

Field, E., \& Harris, D. (1998). A comparative survey of the utility of cross-cockpit linkages and autoflight systems' backfeed to the control inceptors of commercial aircraft. Ergonomics, 41(10), 1462-1477. 
Fine, G.A. (1996). Justifying work: occupational rhetorics as resources in restaurant kitchens. Administrative Science Quarterly, 41, 90-115.

Flach, J. (1999). Beyond error: The language of coordination and stability. In E. Carterrette, \& M. Friedman (Eds.), Handbook of perception and cognition: human performance and ergonomics (2nd edition) ( $\mathrm{pp}$. 109-130). San Diego: Academic Press.

Fowler, R. (1999). Improving communications with ATC. Plane and Pilot, 35(1), 70-72.

Gerber, R., \& Lanksherar, C. (Eds.) (2000). Training for a smart workforce. London, New York: Routledge.

Hartel, C., \& Hartel, G. (1995). Controller resource management: what can we learn from aircrews? Washington: Federal Aviation Administration.

Hollnagel, E., Woods, D.D., \& Leveson, N. (Eds.) (2006). Resilience Engineering. Concepts and Precepts. Aldershot, UK: Ashgate.

Howitt, A.M., \& Leonard, H.B. (2006). Katrina and the core challenges of disaster response. The Fletcher Forum of World Affairs, 30(1), 215-221.

Jentsch, F., Barnett, J., Bowers, C.A., \& Salas, E. (1999). Who is flying this plane anyway? What mishaps tell us about crew member role assignment and air crew situation awareness. Human Factors, 4(1), 1-14.

Jones, G. (1995). Organizational theory: text and cases. Reading, Mass: Addison-Wesley.

Kanki, B.G., \& Palmer, M.T. (1993). Communication and crew resource management. In E.L Wiener, B.G. Kanki, \& R.L. Helmrich (Eds.), Cockpit resource management (pp. 99-136). San Diego, London: Academic Press.

Kerns, K. (1999). Human factors in air traffic control/flight deck integration: implications of data-link simulation research. In D.J. Garland, A.J. Wise, \& V.D. Hopkin (Eds.), Handbook of aviation human factors (pp. 519-546). London: Lawrence Erlbaum \& Associates.

Loftus, G.R. (1979). Short-term memory factors in ground controller/pilot communication. Human Factors, 21(2), 169-181.

McCann, C., Baranski, J., Thompson, M., \& Pigeau, R. (2000). On the utility of experiential cross-training for team decision-making under time stress. Ergonomics, 43(8), 1095-1110.

McCarthy, J., Healey, P., Wright, P., \& Harrison, M.D. (1997). Accountability of work activity in highconsequence work systems: human error in context. International Journal Human-Computer Studies, 47, 735-766.

McGann, A., Morrow, D., Rodvold, M., \& Mackintosh, M-A. (1998). Mixed-media communication on the flight deck: a comparison of voice, data link, and mixed ATC environments. The International Journal of Aviation Psychology, 8(2), 137-156.

Morrow, D., Rodvold, M., \& Lee, A. (1994). Nonroutine transactions in controller-pilot communication. Discourse Processes, 17, 235-258.

Nardi, B. (1996). Context and consciousness: activity theory and human computer interaction. London: MIT Press.

Orlady, H.W., \& Orlady, L.M. (1999). Human factors in multi-crew flight operations. Aldershot, Brookfield USA, Singapore, Sydney: Ashgate.

Owen, C. (1999). Learning in the workplace: The case of air traffic control. Unpublished PhD dissertation, University of Tasmania.

Owen, C. 2001 The role of organisational context in mediating workplace learning and performance. Computers in Human Behaviour, 17 (5/6), 597-614;

Prinzo, O.V. (2004). Automatic dependent surveillance-broadcast/cockpit display of traffic information: innovations in pilot-managed departures. The International Journal of Aviation Psychology, 14(2), 171. 
Rantanen, E.M., McCarley, J.S., \& Xu, X. (2004). Time delays in air traffic control communication loop: effect on controller performance and workload. The International Journal of Aviation Psychology, 14(4), 369.

Rouilleault, H. (2000). Work organization and information and communication technologies. In K. Rubenson, \& H.G. Schuetze (Eds.), Transition to the knowledge society: policies and strategies for individual participation and learning (pp. 209-218). Canada: UBC Institute for European Studies.

Salas, E., Bowers, C.A., \& Edens, E. (2001). An overview of resource management in organizations: why now? In E. Salas, C.A. Bowers, \& E. Edens (eds), Improving teamwork in organizations: applications of resource management training (pp. 1-5). Mahwah, London: Lawrence Erlbaum.

Salas, E., Stagl, K., \& Burke, S. (2004). 25 years of team effectiveness in organizations: Research themes and emerging needs. In C. Cooper, \& I.T. Robertson, International Review of Industrial and Organizational Psychology, 19, 47-9.

Segal, L.D. (1994). Actions speak louder than words: how pilots use nonverbal information for crew communications. Proceedings of the Human Factors and Ergonomics Society 38 th Annual Meeting, 21-25.

Smith-Jentsch, K., Baker, D., Salas, E., \& Cannon-Bowers, J. (2001). Uncovering the differences in team competency requirements: the case of air traffic control teams. In. E. Salas, C.A. Bowers, \& E. Edens (Eds). Improving teamwork in organizations: applications of resource management training (pp. 294320). Mahwah, London: Lawrence Erlbaum.

Suchman, L. (1993). Technologies of accountability: of lizards and aeroplanes. In G. Button (Ed.). Technology in working order: studies of work, interaction, and technology (pp. 113-126). London, New York: Routledge.

Suchman, L. (2000). Located accountabilities in technology production, Lancaster University: Department of Sociology, 13. http://www.comp.lancs.ac.uk/sociology/soc0391s.html

Victor, B., \& Boynton, A.C. (1998). Invented here: Maximizing your organization's internal growth and profitability. Boston: Harvard Business School.

Virkkunen, J. (2006). Dilemmas in building shared transformative agency. @ctivites, 3(1) 43-66

Wiener, E.L., Kanki, B.G., \& Helmrich, R.L. (Eds.) (1993). Cockpit resource management. San Diego, London: Academic Press, 99-136.

Woods, D. 2006. Essential characteristics of resilience. In E. Hollnagel, D. Woods, \& N. Leveson (Eds.), Resilience engineering. Concepts and precepts (pp. 21-34). Aldershot: Ashgate.

Yule, S., Flin, R., \& Murdy, A. (2007). The role of management and safety climate in preventing risk-taking at work. International Journal of Risk Assessment and Management, 7(2), 137-151.

\section{RÉSUMÉ}

L'objectif de cet article est de contribuer au développement d'une troisième génération de travaux centrés sur la théorie historico-culturelle de l'activité. L'analyse porte sur des sujets (inter)agissants, lorsqu'ils sont impliqués dans un travail conjoint situé aux frontières de leurs systèmes d'activité respectifs. L'article discute, de manière théorique, les problématiques soulevées par ces situations, en analysant les pratiques observées lors d'interactions entre des pilotes et des contrôleurs aériens. Les analyses portent sur la manière dont les pratiques de travail sont médiées conjointement, lorsque des changements surviennent dans des systèmes d'activité en interaction. Elles permettent de montrer de quelle manière un changement apporté aux éléments d'un système d'activité génèrent des tensions et des contradictions, et ce faisant, des opportunités de développement dans les interactions quotidiennes entre les contrôleurs aérien et les pilotes de ligne. Sur cette base, l'article définit des problématiques clés à étudier lorsqu'il s'agit de concevoir des interventions dans ces environnements de travail, et 
contribue au développement d'une troisième génération de recherche sur la théorie historico-culturelle de l'activité.

\section{MoTS CLÉS}

Théorie historico-culturelle de l'activité; coordination entre contrôleurs aérien et pilotes d'avion, collaboration, théorie des organisations.

\section{RESUMEN}

Analizando el trabajo conjunto entre sistemas de actividades. El objetivo de este artículo es realizar un aporte al desarrollo de una tercera generación de teorías de la actividad. Lo hace a través del análisis de sujetos que (inter)actúan comprometidos en un trabajo conjunto realizado en los límites de sus respectivos sistemas de actividades. El artículo explora estos problemas desde una perspectiva teórica y discute las prácticas observadas en una interacción entre pilotos y controladores aéreos en la industria aeronáutica. En este caso, se analiza la manera en que las actividades laborales están conjuntamente mediatizadas a través de cambios que ocurren en sistemas de actividades interactivas. El análisis mostrará cómo los cambios en los elementos de los sistemas de actividades revelan puntos de tensión y de contestación y, por ende, oportunidades para el desarrollo de las interacciones cotidianas entre los controladores aéreos y los pilotos de línea. Al abordar el análisis, el artículo introduce algunas preguntas clave a considerar cuando se diseñan las intervenciones en este tipo de entorno laboral, y contribuye al desarrollo de una teoría de la actividad de tercera generación.

\section{Palabras clave}

Teoría histórico-cultural de la actividad, coordinación tripulación/control aéreo, colaboración, teoría organizacional

\section{RÉFÉRENCEMENT}

C. Owen (2008). Analyser le travail conjoint entre différents systèmes d'activité. Activités, 5 (2) pp. 70-89, http://www.activites.org/v5n2/v5n2.pdf

Article soumis en juillet 2006, accepté pour publication le 3 septembre 2008. 